\title{
MINIMALLY INVASIVE TREATMENT FOR GYNECOMASTIA: ULTRASOUND-GUIDED VACUUM-ASSISTED EXCISION - CASE SERIES
}

Henrique Lima Couto', Carolina Nazareth Valadares¹, Osmar Pellegrini Jr¹, Shirley das Graças Ferreira', Julia Grichtolik Cantagalli Paiva'

${ }^{1}$ Redimasto; Redimama - Belo Horizonte (MG), Brazil.

Introduction: Gynecomastia (GM) is the benign proliferation of breast tissue in men. Its prevalence is of up to $65 \%$ according to age. It can be unilateral or bilateral. The standard surgery is periareolar incision, even though it can lead to asymmetry (AS), anesthetic scar, retraction or necrosis of the nipple-areolar complex (NAC). The vacuum-assisted excision (VAE) is the alternative approach. Objectives: To describe the technique and results of ultrasound-guided VAE for GM. Methods: series of 7 cases of Simon I and II GM, submitted to US-guided VAE (10G needle and ENCOR BD) between December 20, 2018, and October 26, 2019. The cases were assessed considering clinical, laboratory and imaging aspects with mammography (MMG) and US. The pre-surgical consent form was obtained. US-guided VAE was performed with a $3 \mathrm{~mm}$ incision, in the ambulatory, with local anesthesia (2\% lidocaine) and no sedation, in the "fine precision" and "dense breast" modes, in order to leave a $1 \mathrm{~cm}$ flap in the retroareolar region. At the end, a vacuum and/or manual drainage of the cavity was performed to reduce the postoperative hematoma (HMA). In the postoperative period, MMGs were performed 1 and 6 months after the VAE, and the revisions occurred on the $7^{\text {th }}$ and $14^{\text {th }}$ day, and the $1^{\text {st }}, 2^{\text {nd }}$ and $6^{\text {th }}$ months. The patients wore vests for 30 postoperative days. The outcomes were good or excellent when the amount of residual breast tissue was minimum, and AS, retractions, necrosis, anesthetic scar or NAC deviation, absent. The patients filled out a form of satisfaction level and perception of the VAE. Results: Mean age was 26.7 years. Mean time of VAE was 28 minutes. The main complaint regarding VAE was aesthetic discomfort, followed by physical deformity. One patient presented with areola skin tear during the procedure. It was sutured and did not impact the aesthetic result. All patients and surgeons reported excellent or sufficient levels of satisfaction. There were no recurrences or re-approach in 6 months. There was no hemorrhage in the peri or postoperative period, with no open approach. No patient required sedation. They all had histology of GM. No cases of breast deviation, necrosis or NAC retraction, infection, AS our anesthetic scar. No patient reported changes in erection or breast sensitivity. They all presented with hemorrhagic suffusion and hematoma with spontaneous resolution in 30 days, without interfering in the outcome. No intervention or hematoma drainage was necessary in the postoperative period. Conclusion: US-guided VAE for GM is alternative to the conventional surgical treatment in Simon I and II cases, with good or excellent results. It presents low complication rates and high satisfaction rates. It is performed in the ambulatory, without intercurrences. Comparative studies of traditional surgery and VAE should be performed. 